\title{
Artroplastia de ombro: tenotomia do subescapular versus osteotomia do tubérculo menor
}

\section{Shoulder Arthroplasty: Tenotomy of the Subscapularis Tendon versus the Lesser Tuberosity Osteotomy}

\author{
Flávio Oliveira França1,2,3@ José Márcio Alves Freitas ${ }^{1,2,3}$ Carlos Alberto Menezes Mariosa1,2,3 \\ Carlos Eduardo Bastiani ${ }^{1,2,3}$ Glaydson Gomes Godinho ${ }^{1,2,3}$
}

\footnotetext{
${ }^{1}$ Grupo de Cirurgia do Ombro e Cotovelo, Hospital Ortopédico BH, Belo Horizonte, MG, Brasil

${ }^{2}$ Grupo de Cirurgia do Ombro e Cotovelo, Hospital Lifecenter, Belo Horizonte, MG, Brasil

${ }^{3}$ Grupo de Cirurgia do Ombro e Cotovelo, Hospital Belo Horizonte, Belo Horizonte, MG, Brasil
}

Rev Bras Ortop 2020;55(4):463-469.

\begin{abstract}
Endereço para correspondência Flávio de Oliveira França, MD, MSc, Hospital Belo Horizonte e Hospital Lifecenter, Rua Prof. Otávo Coelho de Magalhães, 111 - Mangabeiras, Belo Horizonte, MG, 30210-300, Brasil (e-mail: flavio.franca29@gmail.com).
\end{abstract}

\section{Resumo}

Palavras-chave

- prótese de ombro

- artrose

- manguito rotador

\section{Abstract}

Objetivo Avaliar e comparar a função do músculo subescapular entre os grupos submetidos a tenotomia do subescapular (Grupo A) e osteotomia do tubérculo menor (Grupo B), em pacientes tratados por artroplastia anatômica total e parcial de ombros apresentando osteoartrose primária.

Métodos Estudo retrospectivo de pacientes portadores de osteoartrose primária glenoumeral tratados cirurgicamente com prótese anatômica total ou parcial de ombro, avaliados por exame clínico, exames de imagens, escala visual analógica de dor e escore funcional de Constant e Murley. Foram avaliados 28 pacientes, totalizando uma amostra de 32 ombros operados. O seguimento mínimo foi de 12 meses (média 47,45 meses).

Resultados Nos pacientes submetidos à tenotomia do tendão subescapular, dez obtiveram resultado ultrassonográfico com ruptura de sua espessura total (56\%). A consolidação ocorreu em todos pacientes submetidos a osteotomia do tubérculo menor. Não houve diferença entre os grupos na comparação do teste Lift Off, da força nos testes Belly Press e Bear hug, e no escore de Constant e Murley.

Conclusões Não encontramos diferença entre os grupos A e B na comparação do teste Lift Off, da força nos testes Belly Press e Bear hug, e no escore de Constant e Murley.

Objective To evaluate and compare subscapular muscle function among patients undergoing subscapular tenotomy (Group A) and lesser tuberosity osteotomy (Group $B$ ), in patients treated with total and partial anatomic shoulder arthroplasty for primary osteoarthrosis.

Methods Retrospective study of patients with primary glenohumeral osteoarthrosis surgically undergoing total or partial anatomic shoulder prosthesis implant, evaluated by clinical examination, imaging exams, analogue pain scale and Constant and Murley recebido

10 de Abril de 2018

aceito

12 de Fevereiro de 2019
DOI https://doi.org/

10.1055/s-0039-3402469. ISSN 0102-3616.
Copyright $\odot 2020$ by Sociedade Brasileira License terms de Ortopedia e Traumatologia. Published by Thieme Revinter Publicações Ltda, Rio de Janeiro, Brazil 

Keywords
- shoulder prosthesis
- arthrosis
- rotator cuff

functional score. A total of 28 patients were evaluated, totalizing a sample of 32 operated shoulders. The minimum follow-up was of 12 months (mean 47.45 months). Results Among patients submitted to subscapularis tendon tenotomy, 10 had an ultrasound with total rupture of its thickness (56\%). All of the patients of the group B showed lesser tuberosity healing. There was no difference between groups comparing strength evaluated by Belly press and Bear hug tests as well as clinical outcome, through the Constant and Murley score.

Conclusions We did not find differences between Groups A and B evidenciated by comparing strength in the Lift-off test, in the Belly press and Bear hug tests and through he Constant and Murley score.

\section{Introdução}

A prótese anatômica de ombro é uma alternativa de tratamento importante para os casos avançados de degeneração articular, principalmente na osteoartrose $(\mathrm{OA})$ primária, com bons resultados funcionais e alívio da dor. ${ }^{1} \mathrm{Na}$ abordagem cirúrgica deltopeitoral, a mobilização do tendão do músculo subescapular é necessária para adequada exposição da articulação. Tradicionalmente, o manuseio do tendão do subescapular por meio de tenotomia tem sido utilizado, porém com resultados conflitantes quanto à adequada cicatrização e resistência do tendão, assim como função no pós-operatório. ${ }^{2-4}$ Gerber et al. ${ }^{5}$ propuseram e descreveram a técnica de osteotomia do tubérculo menor com a hipótese de que a consolidação óssea (cicatrização osso-osso) diminuiria a possibilidade de complicações e disfunção do subescapular no pós-operatório. ${ }^{5,6}$ No entanto, estudos de revisão não têm consenso sobre a melhor técnica a ser utilizada. ${ }^{7-9}$

O objetivo do presente estudo é avaliar e comparar a função pós-operatória do músculo subescapular nos pacientes submetidos à tenotomia deste tendão ou a osteotomia do tubérculo menor, nas artroplastias anatômicas parciais e totais do ombro. Paralelamente, objetivamos avaliar a integridade do tendão do músculo subescapular nos pacientes submetidos a tenotomia, e a consolidação do tubérculo menor em pacientes operados com a técnica de osteotomia.

\section{Materiais e Métodos}

Trata-se de estudo retrospectivo de pacientes portadores de OA primária glenoumeral tratados com prótese anatômica total ou parcial, por seis ortopedistas especialistas em ombro utilizando a tenotomia do tendão do músculo subescapular (Grupo A) ou a osteotomia do tubérculo menor (Grupo B). O presente estudo foi aprovado pelo Comitê de Ética e Pesquisa sob o número CAAE 83162017.7.0000.5126. Os pacientes que concordaram em participar do estudo assinaram o Termo de Consentimento Livre e Esclarecido.

Os critérios de inclusão foram: pacientes com OA primária tratados cirurgicamente com prótese anatômica total ou parcial de ombro com seguimento mínimo de 12 meses. Foram excluídos do trabalho os pacientes com OA secundária, fratura de úmero proximal, infecção pós-operatória, incapazes de responder os questionários de avaliação funcional ou de comparecerem para reavaliação ambulatorial.

\section{Técnica Operatória}

O paciente foi posicionado em "cadeira de praia," submetido à anestesia geral e bloqueio de plexo braquial. Acesso deltopeitoral, tenotomia e tenodese do tendão da cabeça longa do bíceps junto ao tendão do peitoral maior.

No Grupo A, foram identificados os limites da inserção do tendão do subescapular no tubérculo menor, foi realizada a tenotomia de toda sua extensão crânio-caudal, um centímetro medial à goteira bicipital, e fixação final com fios inabsorvíveis de alta resistência com pontos tendão-tendão.

No Grupo B, o procedimento foi realizado conforme descrição de Gerber et al. ${ }^{10}$ com o uso do osteótomo posicionado paralelo e medial ao sulco bicipital, foi realizada a osteotomia no sentido craniocaudal do intervalo rotador até os vasos circunflexos anteriores, em direção ao limite medial entre a superfície articular e a inserção capsular. Objetiva-se um fragmento ósseo de cerca de $3 \mathrm{a} 4 \mathrm{~cm}$ de comprimento vertical, 5 a $8 \mathrm{~mm}$ de comprimento mediolateral e 5 a $8 \mathrm{~mm}$ de espessura. Foi realizada fixação anatômica do fragmento ósseo com fios inabsorvíveis de alta resistência passados na interface entre tendão-osso e fixados com pontos intraósseos lateral lateralmente ao sulco bicipital.

\section{Coleta dos Dados da Amostra}

Os pacientes foram convidados para retorno ambulatorial para entrevista, avaliação segundo a escala visual analógica de dor (EAD), ${ }^{11}$ aplicação do questionário clínico-funcional de Constant e Murley, ${ }^{12}$ exame físico, e exames de imagem (radiografias e ultrassonografias). No exame físico foi avaliada a amplitude de movimento ativa e passiva, e os testes específicos para avaliação do músculo subescapular (Lift Off test, Belly Press test e Bear hug test). ${ }^{13-15}$

No Lift Off test, ${ }^{13}$ o dorso da mão do paciente foi posicionado na região lombar ao nível de L3. Foi solicitado então o movimento de rotação medial do ombro afastando a mão do corpo. Pacientes incapacitados de afastar o dorso da mão da região lombar ou de manter ativamente a mão afastada foram considerados como Lift Off test positivo. Pacientes que apresentaram limitação de rotação medial, e que não alcançaram o nível de L3, foram considerados incapazes para realizar o Lift Off test. 
Nos testes Belly Press ${ }^{14}$ e Bear hug ${ }^{15}$ utilizamos uma balança doméstica de mola com carga máxima de $20 \mathrm{~kg}$ Performance Plus, (Performance Plus. Indústria e Comércio Ltda, RJ, Brasil) para avaliar a força do músculo subescapular. No Belly Press, solicitou- se que o paciente colocasse a palma da mão no abdômen mantendo o cotovelo à frente do corpo com punho alinhado em 180 graus e que fizesse movimento de rotação medial pressionando a mão contra o abdômen (-Figura 1). A força foi mensurada com o manguito da balança de mola entre a palma da mão e o abdômen, enquanto o examinador realizava força com aumento progressivo, contrária à de rotação medial do paciente. A força de rotação medial máxima durante o teste foi avaliada no momento em que o paciente não conseguia manter a sustentação da mão junto ao abdômen ou quando não mantinha o cotovelo à frente do tronco, ocasionando a extensão do braço e flexão do punho, indicativo de perda de força da rotação medial. No teste Bear hug, foi solicitado ao paciente posicionar a mão espalmada do lado acometido no ombro contralateral com os dedos estendidos, mantendo o ombro fletido em 90 graus. 0 manguito da balança foi então colocado no antebraço do lado acometido e uma força perpendicular a ele foi realizada pelo examinador, com intuito de descolar a mão do paciente do seu ombro (-Figura 2). Solicitamos ao paciente que resistisse à manobra. A força máxima foi medida no momento em que o paciente não conseguia manter a mão junto ao ombro ou quando não permanecia com o braço fletido em 90 graus, realizando flexão do punho e extensão do ombro.

Todos os pacientes realizaram radiografias de controle nas incidências anteroposterior verdadeiro (em neutro, rotação medial e rotação lateral), e nos perfis axilar e escapular, para avaliar o posicionamento dos implantes e a presença ou não de soltura dos componentes da prótese. No Grupo B, a consolidação óssea foi avaliada pela radiografia em perfil axilar que permite melhor visualização da continuidade da cortical do tubérculo menor e da cabeça umeral $^{5}$ (- Figura 3).

No Grupo A, utilizamos a ultrassonografia para avaliação da integridade do tendão do músculo subescapular, definidos como tendão normal ( - Figura 4A), ruptura de espessura total sem comprometer toda a extensão craniocaudal ou ruptura de

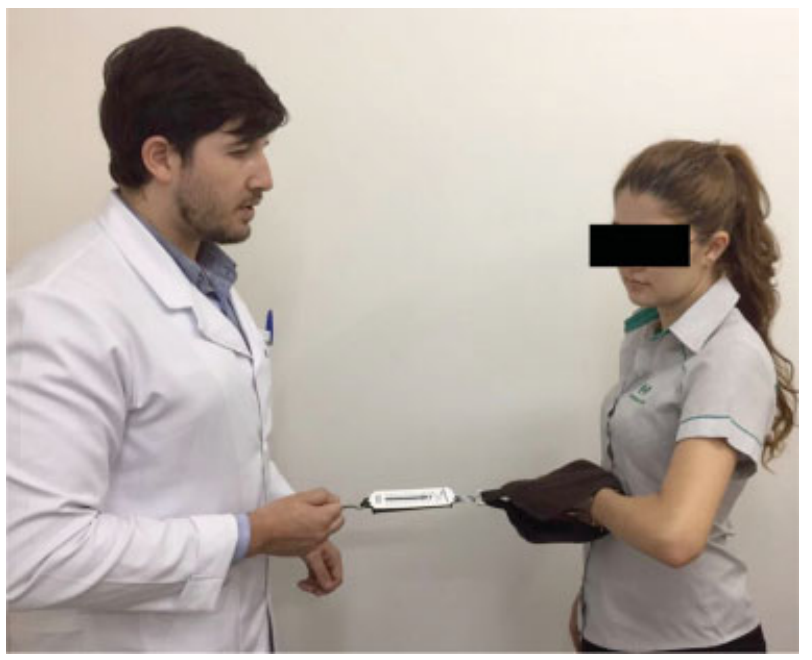

Fig. 1 Aferição da força no teste Belly Press.

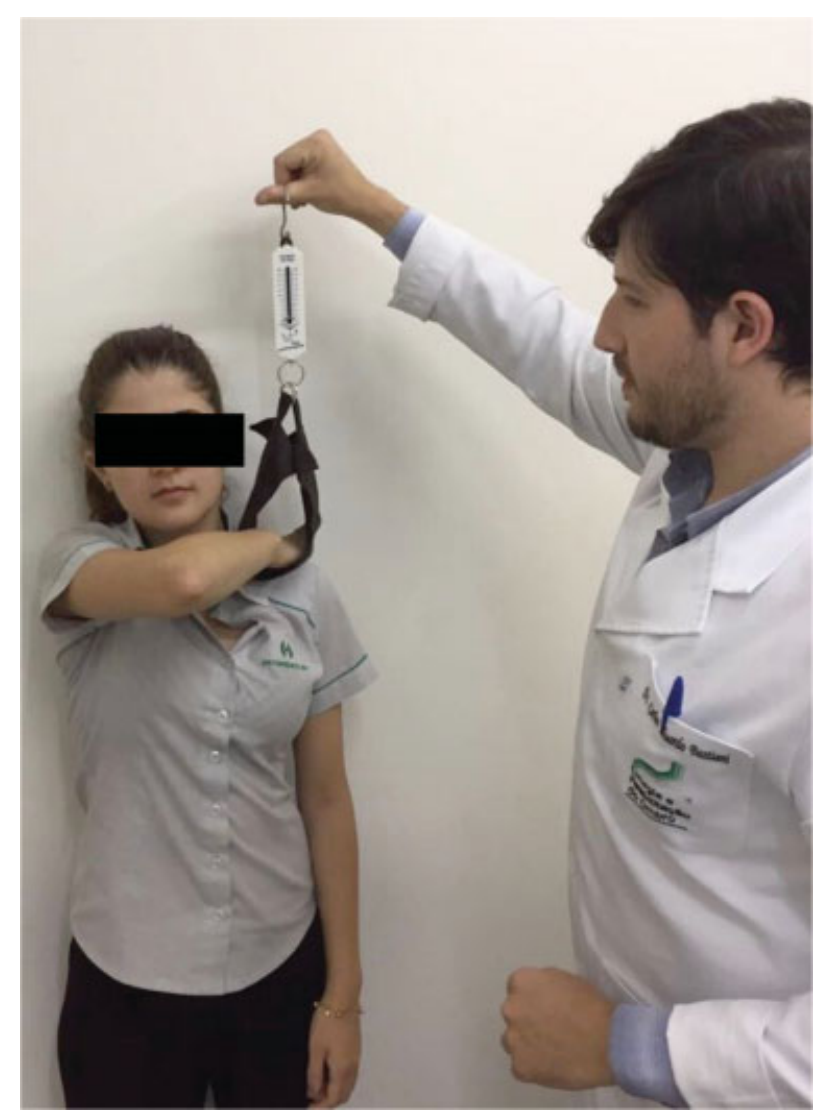

Fig. 2 Avaliação da força no teste Bear hug.

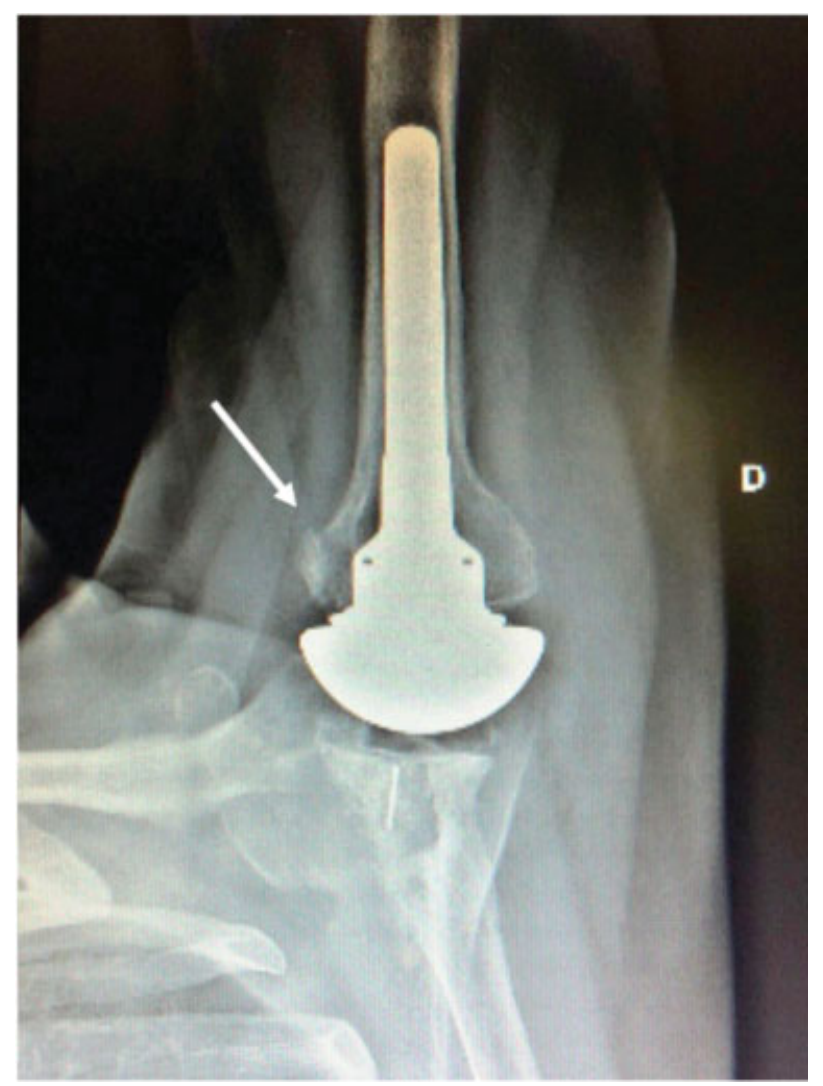

Fig. 3 Radiografia em perfil axilar demonstrando a consolidação do tubérculo menor após artroplastia total de ombro. 

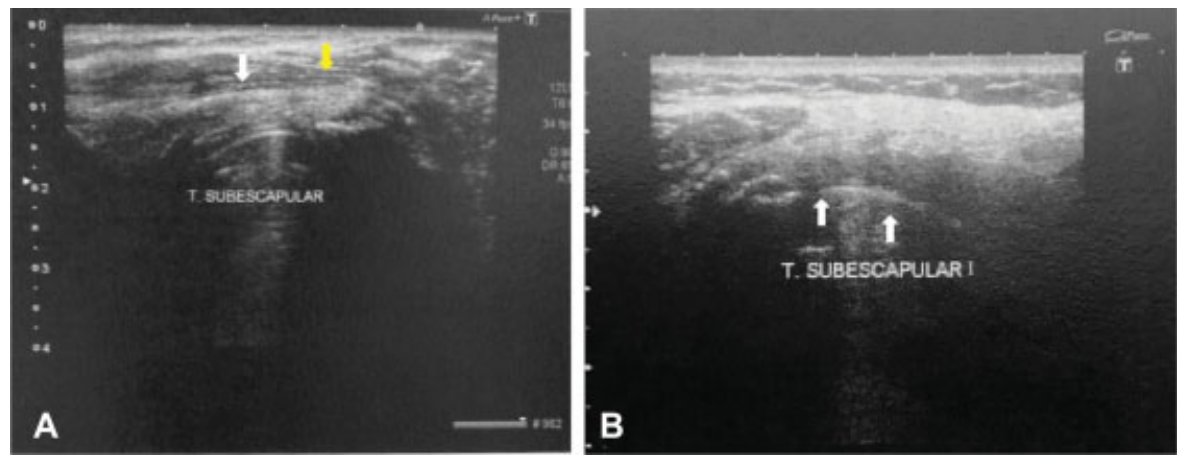

Fig. 4 Ultrassonografia para avaliação da integridade do tendão do músculo subescapular. Legenda: (A) ultrassonografia constatando espessura preservada, contornos regulares e aspecto hipoecogênico habitual do tendão subescapular; (B) tendão não individualizado, notando-se área anecoica em sua topografia.

extensão total comprometendo toda a extensão craniocaudal (-Figura 4B). Todos os exames de ultrassom foram realizados por um mesmo radiologista ultrassonografista com experiência em avaliação músculoesquelética.

Na comparação dos resultados pós-operatórios das técnicas tenotomia e osteotomia, foram utilizados o teste $\mathrm{t}$ de Student para amostras independentes e o teste qui-quadrado ou exato de fisher. Quando não foi encontrada diferença estatística, foi lançado mão da avaliação pelo $d$ de Cohen ${ }^{16}$ (comparação entre médias) e $V$ de Cramer (comparação entre porcentagens), onde tenta-se mostrar o tamanho do efeito dos resultados na prática, por meio de escala de magnitude. Nessa escala, adota-se a classificação: $d$ de Cohen, pequeno 0,20 a 0,49 , moderado 0,50 a 0,79 e elevado $\geq 0,80$ e $v$ de Cramer: pequeno 0,10 a 0,29 ; moderado 0,30 a 0,49 e elevado $\geq 0,50 .^{16}$ Todas as análises foram feitas no IBM SPSS Statistics for Windows, versão 20 (IBM Corp., Armonk, NY, EUA). O valor de $p \leq 0,05$ foi adotado para significância estatística.

\section{Resultados}

Entre maio e novembro de 2017, foram avaliados 28 pacientes que atendiam aos critérios de inclusão, totalizando uma amostra de 32 ombros operados. 0 grupo A foi composto de 16 pacientes ( 18 ombros) e o grupo B de 13 pacientes (14 ombros). A média de idade foi de 72 anos (44-82 anos), sendo a maioria do sexo feminino (75\%). A maioria das cirurgias foi realizada no ombro direito $(n=21 ; 65,6 \%)$. Quatro pacientes (8 ombros) foram submetidos à prótese bilateral (14,2\%), sendo que em 5 ombros foi utilizada a tenotomia,e em 3 a osteotomia. 0 tempo médio de seguimento foi de 3,52 anos (1-11 anos). Artroplastia total foi o procedimento mais realizado (93,3\%). Todos os pacientes tinham diagnóstico de OA primária.

Os dados demográficos relacionados aos grupos estudados estão expressos na - Tabela 1. Não foi observada diferença significativa entre os grupos em relação ao sexo, idade e lateralidade $(p>0,05)$. Porém, o tempo de avaliação pósoperatório foi maior nos pacientes do Grupo A.

Os dados de exames de imagens (radiografias e ultrassonografias) podem ser vistos na -Tabela 2. Todos os pacientes
Tabela 1 Dados demográficos dos pacientes submetidos a tenotomia e a ostetomia

\begin{tabular}{|c|c|c|c|c|}
\hline Variáveis & $\begin{array}{l}\text { Tenotomia } \\
(\mathrm{n}=18) \\
(\%)\end{array}$ & $\begin{array}{l}\text { Osteotomia } \\
(n=14) \\
(\%)\end{array}$ & valor-p & $\mathrm{TE}$ \\
\hline \multicolumn{5}{|l|}{ Gênero } \\
\hline Feminino & $12(67,0)$ & $12(86,0)$ & 0.410 & 0.22 \\
\hline Masculino & $6(33,0)$ & $2(14,0)$ & & \\
\hline Idade (anos) & $72,4 \pm 6,5$ & $71,6 \pm 9,2$ & 0.770 & 0.10 \\
\hline \multicolumn{5}{|l|}{ Lateralidade } \\
\hline Direito & $11(61,0)$ & $6(43,0)$ & \multirow[t]{3}{*}{0.310} & \multirow[t]{3}{*}{0.30} \\
\hline Esquerdo & $2(11,0)$ & $5(36,0)$ & & \\
\hline Ambos & $5(28,0)$ & $3(21,0)$ & & \\
\hline $\begin{array}{l}\text { Acompanhamento } \\
\text { (meses) }\end{array}$ & $62 \pm 38$ & $22 \pm 8$ & $0,001^{*}$ & - \\
\hline
\end{tabular}

Abreviação: TE, tamanho do efeito.

Média \pm desvio-padrão para variáveis quantitativas.

Frequência absoluta (\%) para variáveis qualitativas.

Tabela 2 Resultado da avaliação radiográfica e ultrassonográfica dos pacientes submetidos à tenotomia e osteotomia

\begin{tabular}{|l|l|l|l|l|l|}
\hline & $\begin{array}{l}\text { Tenotomia } \\
\text { (Grupo A) } \\
(\mathbf{n}=\mathbf{1 8})(\%)\end{array}$ & $\begin{array}{l}\text { Osteotomia } \\
\text { (Grupo B) } \\
(\mathbf{n}=\mathbf{1 4})(\%)\end{array}$ & valor-p & TE \\
\hline Radiografia & $18(100,0)$ & $14(100,0)$ & - & - \\
\hline $\begin{array}{l}\text { Tuberculo menor } \\
\text { normoposicionado }\end{array}$ & 18 & & - \\
\hline USG & $0(0,0)$ & $1(12,0)$ & - & - \\
\hline Ruptura Parcial & $10(56,0)$ & $2(25,0)$ & - & - \\
\hline Ruptura Total & & & &
\end{tabular}

Abreviação: TE, tamanho do efeito.

Média \pm desvio-padrão para variáveis quantitativas.

Frequência absoluta (\%) para variáveis qualitativas.

do grupo B conseguiram realizar a radiografia em axilar e todos apresentaram consolidação do tubérculo menor. Destaca-se que 56\% dos pacientes do Grupo A apresentaram ruptura de espessura total do tendão subescapular. 
A - Tabela 3 apresenta os resultados clínicos e funcionais. Não foram observadas diferenças estatisticamente significativas entre os Grupos A e B em relação a esses indicadores $(p>0,05)$.

$\mathrm{Na}-$ Tabela 4 estão expostos os dados correlacionados ao escore funcional de Constant e Murley (valor total e ponderado), onde não se vê diferença estatisticamente significativa.

$\mathrm{Na}$ - Tabela 5 estão os resultados da comparação dos achados ultrassonográficos dos pacientes do Grupo A em relação ao exame físico e ao escore de Constant e Murley total e ponderado. Os pacientes com ruptura total do tendão do subescapular apresentaram menor elevação anterior ativa (EAA) quando comparados aos pacientes que apresentaram tendão normal $(p=0,03)$ (-Figura 5 ).

Tabela 3 Comparação do resultado pós-operatório clínico dos pacientes submetidos a tenotomia e a osteotomia

\begin{tabular}{|c|c|c|c|c|}
\hline Variáveis & $\begin{array}{l}\text { Tenotomia } \\
(G \text { rupo } A) \\
(n=18)\end{array}$ & $\begin{array}{l}\text { Osteotomia } \\
\text { (Grupo B) } \\
(\mathrm{n}=14)\end{array}$ & valor-p & TE \\
\hline$E A A$ & $147 \pm 40$ & $138 \pm 37$ & 0.46 & - \\
\hline$E A P$ & $165 \pm 26$ & $156 \pm 24$ & 0.13 & - \\
\hline RL1 ativa & $69 \pm 11$ & $61 \pm 15$ & 0.12 & - \\
\hline RL1 passiva & $69 \pm 11$ & $62 \pm 16$ & 0.21 & - \\
\hline RL2 ativa & $77 \pm 12$ & $80 \pm 13$ & 0.34 & - \\
\hline RL2 passiva & $82 \pm 9$ & $81 \pm 13$ & 0.90 & - \\
\hline \multicolumn{5}{|l|}{ Rotação Medial } \\
\hline T7 a T12 & $14(78,0)$ & $13(93,0)$ & 0.61 & 0.24 \\
\hline L1 a L5 & $2(11,0)$ & $0(0,0)$ & & \\
\hline Glúteo & $2(11,0)$ & $1(7,0)$ & & \\
\hline \multicolumn{5}{|l|}{ Lift-Off } \\
\hline Não realiza & $3(17,0)$ & $0(0,0)$ & \multirow[t]{3}{*}{0.23} & \multirow[t]{3}{*}{0.32} \\
\hline Negativo & $11(61,0)$ & $11(86,0)$ & & \\
\hline Positivo & $4(22,0)$ & $3(14,0)$ & & \\
\hline $\begin{array}{l}\text { Belly Press } \\
\text { ( força) }\end{array}$ & $5,3 \pm 2,0$ & $5,0 \pm 2,5$ & 0.73 & 0.14 \\
\hline $\begin{array}{l}\text { Bear Hug } \\
\text { (força) }\end{array}$ & $4,9 \pm 1,4$ & $4,6 \pm 2,8$ & 0.72 & 0.14 \\
\hline
\end{tabular}

Abreviações: EAA, Elevação anterior ativa; EAP, elevação anterior passiva; RE2, Rotação lateral 2; RL1, rotação lateral 1; TE, tamanho do efeito). Média \pm desvio-padrão para variáveis quantitativas. Frequência absoluta (\%) para variáveis qualitativas.

Tabela 4 Comparação do Escore de Constant total e ponderado dos pacientes submetidos à tenotomia e osteotomia

\begin{tabular}{|l|l|l|l|l|}
\hline Variáveis & $\begin{array}{l}\text { Tenotomia } \\
(\text { Grupo A) } \\
(\mathbf{n}=\mathbf{1 8})\end{array}$ & $\begin{array}{l}\text { Osteotomia } \\
(\text { Grupo B) } \\
(\mathbf{n}=14)\end{array}$ & valor-p & TE \\
\hline $\begin{array}{l}\text { Constant } \\
\text { (total) }\end{array}$ & $72 \pm 13$ & $75 \pm 15$ & 0.56 & 0.21 \\
\hline $\begin{array}{l}\text { Constant } \\
\text { (total } \\
\text { ponderado) }\end{array}$ & $85 \pm 13$ & $90 \pm 18$ & 0.43 & 0.32 \\
\hline
\end{tabular}

Abreviação: TE, tamanho do efeito.

Média \pm desvio-padrão para variáveis quantitativas.

Frequência absoluta (\%) para variáveis qualitativas.
Tabela 5 Comparação do resultado pós-operatório clínico e funcional de pacientes submetidos à tenotomia ( Grupo A) com e sem ruptura do tendão subescapular

\begin{tabular}{|c|c|c|c|c|}
\hline Variáveis & $\begin{array}{l}\text { Sem } \\
\text { Ruptura } \\
(n=8) \\
(\%)\end{array}$ & $\begin{array}{l}\text { Ruptura } \\
\text { Total } \\
(n=10) \\
(\%)\end{array}$ & valor-p & TE \\
\hline $\begin{array}{l}\text { Escala Analógica } \\
\text { de Dor }\end{array}$ & $1,0 \pm 1,2$ & $1,6 \pm 2,9$ & 0.39 & - \\
\hline Belly & $6,0 \pm 3,0$ & $5,0 \pm 1,0$ & 0.27 & 0.50 \\
\hline Bear Hug & $5,0 \pm 2,0$ & $4,9 \pm 1,0$ & 0.89 & 0.07 \\
\hline \multicolumn{5}{|l|}{ Lift-Off } \\
\hline Não realiza & $1(13,0)$ & $2(20,0)$ & \multirow[t]{3}{*}{1.00} & \multirow[t]{3}{*}{0.11} \\
\hline Negativo & $5(62,0)$ & $6(60,0)$ & & \\
\hline Positivo & $2(25,0)$ & $2(20,0)$ & & \\
\hline \multicolumn{5}{|l|}{ Rotação Medial } \\
\hline T7 a T12 & $6(75,0)$ & $8(80,0)$ & \multirow[t]{3}{*}{1.00} & \multirow[t]{3}{*}{0.06} \\
\hline L1 a L5 & $1(12,5,0)$ & $1(10,0)$ & & \\
\hline Glúteo & $1(12,5,0)$ & $1(10,0)$ & & \\
\hline$E A A$ & $168 \pm 24$ & $130 \pm 43$ & $0,03^{*}$ & - \\
\hline$E A P$ & $174 \pm 14$ & $158 \pm 30$ & 0.14 & - \\
\hline RL1 ativa & $71 \pm 8$ & $67 \pm 12$ & 0.31 & - \\
\hline RL1 passiva & $71 \pm 10$ & $67 \pm 12$ & 0.38 & - \\
\hline RL2 ativa & $80 \pm 11$ & $75 \pm 13$ & 0.38 & - \\
\hline RL2 passiva & $82 \pm 10$ & $82 \pm 9$ & 0.96 & - \\
\hline $\begin{array}{l}\text { Constant e } \\
\text { Murley (total) }\end{array}$ & $77 \pm 15$ & $68 \pm 9$ & 0.12 & 0.75 \\
\hline $\begin{array}{l}\text { Constant e Murley } \\
\text { (total ponderado) }\end{array}$ & $91 \pm 14$ & $81 \pm 10$ & 0.12 & 0.83 \\
\hline
\end{tabular}

Abbreviations: EAA, Elevação anterior ativa; EAP, elevação anterior passiva; RE2, Rotação lateral 2; RL1, rotação lateral 1; TE, tamanho do efeito.

Média \pm desvio-padrão para variáveis quantitativas.

Frequência absoluta (\%) para variáveis qualitativas.

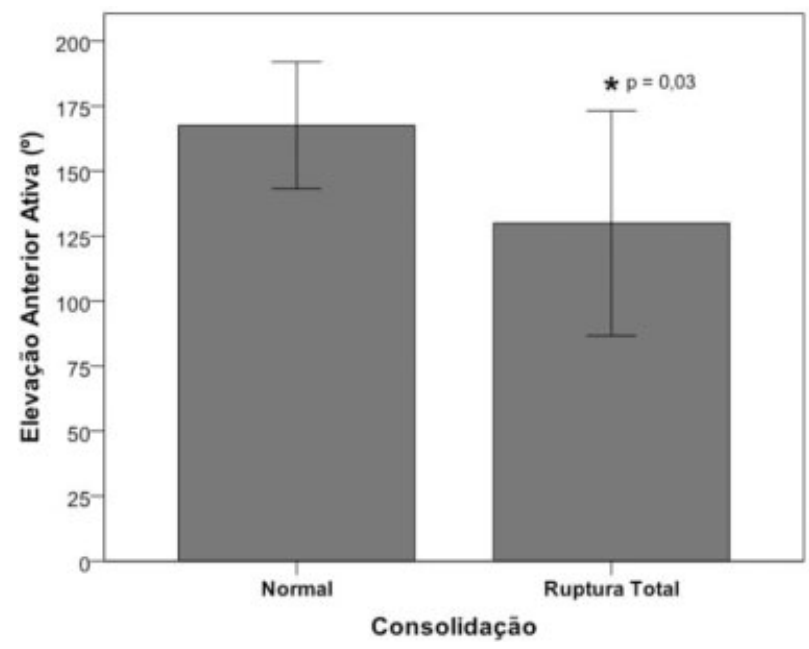

Fig. 5 Elevação anterior ativa de pacientes submetidos a tenotomia que apresentaram consolidação normal $(n=8)$ e ruptura total do tendão $(n=10)$. Legenda: *diferença estatisticamente significativa, $p<0,05$. 
As complicações ocorreram em cinco procedimentos (15,62\%), sendo quatro no Grupo A e uma no Grupo B. A soltura asséptica do implante foi a complicação mais comum no Grupo A (dois casos), seguido por luxação posterior da cabeça (um caso) e fratura periprotética (um caso). No Grupo B, apenas um paciente teve como complicação a ruptura do tendão subescapular com consequente diminuição do espaço úmero-acromial, sem soltura do implante.

\section{Discussão}

Desde 1982, com a publicação de Neer $^{17}$ a respeito dos excelentes desfechos funcionais da artroplastia de ombro para OA primária em pacientes sem lesão do manguito rotador, e posteriormente com o desenvolvimento da terceira geração de próteses de ombro, os resultados cirúrgicos apresentaram melhora significativa. A sobrevida da prótese de ombro nos modelos atuais é de $97 \%$ em 10 anos e $84 \%$ em 20 anos, com $<10 \%$ de complicações em 10 anos. ${ }^{17}$ Em nosso estudo, para minimizar o risco de falha de implantes e objetivando melhor resultado funcional, todas as cirurgias foram realizadas com máximo rigor técnico quanto ao posicionamento dos implantes, não havendo diferença entre os grupos.

Em nosso estudo, encontramos um perfil epidemiológico semelhante aos outros da literatura, ${ }^{8,18}$ com predominância de mulheres no grupo A de $67 \%$ e no grupo B de $86 \%$, média de idade de 72 anos e tempo médio de seguimento de 3,5 anos. Observamos resultados semelhantes entre os grupos em relação ao sexo, idade e lateralidade $(p>0,05)$. Porém, o tempo de avaliação pós-operatório foi menor nos pacientes submetidos à osteotomia do tubérculo menor, pois a mesma começou a ser utilizada posteriormente à técnica de tenotomia do tendão do subescapular, o que pode ser considerado um viés de avaliação. Da mesma forma, apesar da técnica cirúrgica ser idêntica entre os seis cirurgiões nos respectivos grupos de pacientes, pois todos trabalham juntos e obtiveram a mesma formação técnica, não podemos deixar de citar esse possível viés no resultado cirúrgico dos pacientes.

A baixa pontuação na EAD no período pós-operatório dos nossos pacientes demonstra o excelente resultado da artroplastia para o alívio da dor. Em nosso estudo, a média da EAD foi $<1,2$ pontos nos dois grupos, indo ao encontro do que apresenta a literatura mundial. ${ }^{18}$

No presente estudo, os pacientes apresentaram elevação anterior, do grupo tenotomia, e rotação lateral junto ao corpo, em ambos os grupos, com valores superiores ao apresentado por Jandhyala et al. ${ }^{19}$ Por outro lado, os mesmos autores apresentaram uma média de elevação ativa para o grupo de osteotomia superior ao nosso trabalho.

Com relação à medida da força, nos testes Belly Press e Bear hug, os grupos apresentaram resultados semelhantes, fato este encontrado também por outros autores. ${ }^{20,21}$ Da mesma forma, Buckley et al. ${ }^{21}$ observaram que a força nos testes Belly Press e Bear hug tendeu a ser maior no grupo osteotomia, mas sem diferença estatística. Na execução do Lift Off Test, nosso estudo demonstrou que todos os pacientes do Grupo B conseguiram realizá-lo, sendo que dois apresentaram teste positivo. Três pacientes no Grupo A não conseguiram realizar o teste devido ao déficit de rotação medial, e quatro apresentaram teste positivo. Não houve diferença estatística entre os grupos. Caplan et al. ${ }^{2}$ avaliando 45 tenotomias, obtiveram Lift off negativo em 41 pacientes, quatro inconclusivos porque não conseguiam alcançar a mão até a região lombar e Bear hug negativo nos 45 ombros.

No presente estudo, o grupo tenotomia apresentou ruptura total do tendão do subescapular em $56 \%$ dos ombros operados. Esse achado assemelha-se aos de outros autores ${ }^{18,20-22}$ que tiveram alterações na avaliação ultrassonográfica do tendão. Em nosso estudo, observamos que todos os pacientes do Grupo B tiveram consolidação do tubérculo menor, o que acompanhou uma tendência da literatura. ${ }^{19,20,23}$

No Grupo B, percebemos que três pacientes apresentaram resultados clínicos ruins durante o exame clínico (Lift Off positivo e pouca força nos testes Belly Press e Bear hug). Devido a isso, optamos por solicitar ultrassom para avaliar a integridade do tendão do músculo subescapular e o resultado foi de ruptura total em dois desses pacientes e ruptura parcial em um. Acreditamos que, como não foi abordada durante $o$ ato cirúrgico, a ruptura tendínea tenha ocorrido por outros motivos não relacionados à técnica, o que pode ter piorado os resultados do grupo. Em um desses pacientes com ruptura total, identificamos diminuição da distância úmeroacromial, sugerindo lesão extensa do manguito.

Quanto ao teste de avaliação funcional de Constant e Murley, encontramos resultados satisfatórios e semelhantes entre os grupos tenotomia (total $=72$, ponderado $=85$ ) e osteotomia (total $=75$, ponderado $=90$ ). Buckley et al. ${ }^{21}$ não obtiveram diferença entre os grupos. Liem et al. ${ }^{22}$ na avaliação de 23 pacientes operados por desinserção subescapular, o escore de Constant e Murley nos pacientes com Lift Off test positivo foi de 43,8, e 62,7 naqueles com Lift Off test negativo, ambos abaixo dos valores encontrados em nossa amostra. Para Qureshi et al. ${ }^{23}$ na avaliação de 30 artroplastias com osteotomias do tubérculo menor, o escore de Constant e Murley foi de 64, também abaixo dos nossos valores.

Realizamos também análise no grupo A entre os pacientes que apresentaram ruptura total $(n=10)$ e os que tinham tendão íntegro $(n=8)$. Ao avaliar o escore de Constant e Murley, não observamos diferença estatística. No entanto, nos pacientes sem ruptura, o tamanho do efeito ( $d$ de Cohen) foi alto $(d=0,83)$. Do ponto de vista prático, isso demonstra uma elevada magnitude para melhor resultado do escore no grupo sem ruptura. Não houve diferença nos testes Lift Off, Bear hug e Belly Press entre esses pacientes; no entanto, o tamanho do efeito foi moderado $(\mathrm{d}=0,50)$ no Belly Press para o grupo sem ruptura, indicando uma magnitude moderada para maior força nesse teste nos pacientes com tendão normal na ultrassonografia. Observamos que os pacientes com o tendão do subescapular íntegro apresentaram EAA significativamente maior do que aqueles com lesão total $(p=0,03)$, fato esse que pode ser explicado pela perda do balanço de forças anterior e posterior do manguito rotador.

Os resultados semelhantes entre os grupos nos testes específicos do músculo subescapular, mesmo em vigência da grande incidência de ruptura total do tendão do subescapular no grupo tenotomia, pode ser explicado pela grande 
variabilidade encontrada na literatura quanto à sensibilidade e especificidade dos testes, o que poderia justificar a quantidade de falsos negativos. ${ }^{15,24,25}$

\section{Conclusões}

No presente estudo, os pacientes submetidos a artroplastia anatômica total ou parcial de ombro por meio da tenotomia do músculo subescapular ou da osteotomia do tubérculo menor apresentam resultados clíncos, radiográficos e funcionais semelhantes.

\section{Conflito de Interesses}

Os autores declaram não haver conflito de interesses.

\section{Referências}

1 Cofield RH. Total shoulder arthroplasty with the Neer prosthesis. J Bone Joint Surg Am 1984;66(06):899-906

2 Caplan JL, Whitfield B, Neviaser RJ. Subscapularis function after primary tendon to tendon repair in patients after replacement arthroplasty of the shoulder. J Shoulder Elbow Surg 2009;18(02): 193-196, discussion 197-198

3 Miller SL, Hazrati Y, Klepps S, Chiang A, Flatow EL. Loss of subscapularis function after total shoulder replacement: A seldom recognized problem. J Shoulder Elbow Surg 2003;12(01): 29-34

4 Miller BS, Joseph TA, Noonan TJ, Horan MP, Hawkins RJ. Rupture of the subscapularis tendon after shoulder arthroplasty: diagnosis, treatment, and outcome. J Shoulder Elbow Surg 2005;14(05): 492-496

5 Gerber C, Yian EH, Pfirrmann CA, Zumstein MA, Werner CM. Subscapularis muscle function and structure after total shoulder replacement with lesser tuberosity osteotomy and repair. J Bone Joint Surg Am 2005;87(08):1739-1745

6 Van den Berghe GR, Nguyen B, Patil S, et al. A biomechanical evaluation of three surgical techniques for subscapularis repair. J Shoulder Elbow Surg 2008;17(01):156-161

7 Schrock JB, Kraeutler MJ, Houck DA, Provenzano GG, McCarty EC, Bravman JT. Lesser tuberosity osteotomy and subscapularis tenotomy repair techniques during total shoulder arthroplasty: A meta-analysis of cadaveric studies. Clin Biomech (Bristol, Avon) 2016;40:33-36

8 Louie PK, Levy DM, Bach BR Jr, Nicholson GP, Romeo AA. Subscapularis Tenotomy Versus Lesser Tuberosity Osteotomy for Total Shoulder Arthroplasty: A Systematic Review. Am J Orthop 2017;46(02):E131-E138

9 Choate WS, Kwapisz A, Momaya AM, Hawkins RJ, Tokish JM. Outcomes for subscapularis management techniques in shoulder arthroplasty: a systematic review. J Shoulder Elbow Surg 2018;27 (02):363-370
10 Gerber C, Pennington SD, Yian EH, Pfirrmann CA, Werner CM, Zumstein MA. Lesser tuberosity osteotomy for total shoulder arthroplasty. Surgical technique. J Bone Joint Surg Am 2006;88 (Suppl 1 Pt 2):170-177

11 Jensen MP, Karoly P, Braver S. The measurement of clinical pain intensity: a comparison of six methods. Pain 1986;27(01): 117-126

12 Constant CR, Gerber C, Emery RJ, Søjbjerg JO, Gohlke F, Boileau P. A review of the Constant score: modifications and guidelines for its use. J Shoulder Elbow Surg 2008;17(02):355-361

13 Gerber C, Krushell RJ. Isolated rupture of the tendon of the subscapularis muscle. Clinical features in 16 cases. J Bone Joint Surg Br 1991;73(03):389-394

14 Gerber C, Hersche O, Farron A. Isolated rupture of the subscapularis tendon. J Bone Joint Surg Am 1996;78(07):1015-1023

15 Barth JR, Burkhart SS, De Beer JF. The bear-hug test: a new and sensitive test for diagnosing a subscapularis tear. Arthroscopy 2006;22(10):1076-1084

16 Cohen J. A power primer. Psychol Bull 1992;112(01):155-159

17 Walch G, Boileau P, Noël E. Shoulder arthroplasty: evolving techniques and indications. Joint Bone Spine 2010;77(06): 501-505

18 Shafritz AB, Fitzgerald MG, Beynnon BD, DeSarno MJ. Lift-off Test Results After Lesser Tuberosity Osteotomy Versus Subscapularis Peel in Primary Total Shoulder Arthroplasty. J Am Acad Orthop Surg 2017;25(04):304-313

19 Jandhyala S, Unnithan A, Hughes S, Hong T. Subscapularis tenotomy versus lesser tuberosity osteotomy during total shoulder replacement: a comparison of patient outcomes. J Shoulder Elbow Surg 2011;20(07):1102-1107

20 Scalise JJ, Ciccone J, Iannotti JP. Clinical, radiographic, and ultrasonographic comparison of subscapularis tenotomy and lesser tuberosity osteotomy for total shoulder arthroplasty. J Bone Joint Surg Am 2010;92(07):1627-1634

21 Buckley T, Miller R, Nicandri G, Lewis R, Voloshin I. Analysis of subscapularis integrity and function after lesser tuberosity osteotomy versus subscapularis tenotomy in total shoulder arthroplasty using ultrasound and validated clinical outcome measures. J Shoulder Elbow Surg 2014;23(09):1309-1317

22 Liem D, Kleeschulte K, Dedy N, Schulte TL, Steinbeck J, Marquardt B. Subscapularis function after transosseous repair in shoulder arthroplasty: transosseous subscapularis repair in shoulder arthroplasty. J Shoulder Elbow Surg 2012;21(10):1322-1327

23 Qureshi S, Hsiao A, Klug RA, Lee E, Braman J, Flatow EL. Subscapularis function after total shoulder replacement: results with lesser tuberosity osteotomy. J Shoulder Elbow Surg 2008;17(01): $68-72$

24 Itoi E, Minagawa H, Yamamoto N, Seki N, Abe H. Are pain location and physical examinations useful in locating a tear site of the rotator cuff? Am J Sports Med 2006;34(02):256-264

25 Leroux JL, Thomas E, Bonnel F, Blotman F. Diagnostic value of clinical tests for shoulder impingement syndrome. Rev Rhum Engl Ed 1995;62(06):423-428 